\title{
Evaluación del Éxito de Tratamientos Endodónticos Realizados por Estudiantes de Pregrado en una Universidad Chilena
}

\author{
Evaluation of Success Rate of Root Canal Treatment Performed by \\ Undergraduate Dental Students in a Chilean University
}

Felipe Maldonado-Sanhueza1; Vania Gómez-Inzunza'1,2; Cristián Rosas-Mendez ${ }^{3}$ \& Scarlette Hernández-Vigueras S1,3 $^{1}$

MALDONADO-SANHUEZA, F.; GÓMEZ-INZUNZA, V.; ROSAS-MENDEZ, C. \& HERNÁNDEZ-VIGUERAS, S. Evaluación del éxito de tratamientos endodónticos realizados por estudiantes de pregrado en una Universidad chilena. Int. J. Odontostomat., 14(2):154-159, 2020.

RESUMEN: El tratamiento endodóntico tiene como finalidad prevenir o lograr la ausencia de periodontitis apical post-tratamiento, logrando la mantención del diente en la boca de manera funcional. La realización de tratamientos endodónticos de baja complejidad en la formación de pregrado es transversal a todas las universidades chilenas. El objetivo del presente estudio es evaluar la frecuencia de éxito de tratamientos endodónticos realizados por estudiantes de pregrado entre los años 2014-2017 de la Escuela de Odontología, Universidad Austral de Chile (UACh), Valdivia, Chile. Se realizó un estudio observacional descriptivo donde fueron evaluados 74 tratamientos endodónticos en dientes uniradiculares de 63 pacientes seleccionados a través de un muestreo aleatorio simple, que cumplieran con los criterios de selección. Los parámetros usados para la evaluación clínica fueron: ausencia de dolor a la palpación y percusión, ausencia de fístula y diente en función. En la evaluación radiográfica se utilizaron los siguientes parámetros: longitud de obturación en relación al ápice dentario, conicidad, densidad del material obturador y reducción de la lesión periapical. De los tratamientos evaluados se reportó un $78 \%$ de éxito clínico y un $41 \%$ de éxito radiográfico. Finalmente, se concluye que los resultados obtenidos a nivel clínico y radiográfico son similares a los reportados en la literatura.

PALABRAS CLAVE: endodoncia, radiografía dental, estudiantes de odontología, obturación radicular, fracaso, éxito, dolor.

\section{INTRODUCCIÓN}

El tratamiento endodóntico tiene como finalidad prevenir o lograr la ausencia de periodontitis apical postratamiento y mantener el diente tratado en función (Marquis et al., 2006; Wu et al., 2009). Consta de diversas etapas, como el acceso, la remoción de tejido pulpar, desinfección, conformación de los conductos y la obturación final (Sjogren et al. 1990).

Una obturación de alta calidad de los conductos es uno de los indicadores de un tratamiento endodóntico exitoso (Sjörgen et al.). Para lograr este tipo de obturación debe ser realizada de forma ade- cuada, homogénea y tridimensional para prevenir la percolación y microfiltración hacia los tejidos periapicales y también en sentido contrario (Ray \& Trope, 1995; American Association of Endodontists, 1998). Sin embargo, una pobre obturación sigue siendo un hecho habitual en la práctica dental, en particular en estudiantes de pregrado (Sjörgen et al.) debido a algunas dificultades o factores que pueden presentarse durante el tratamiento e inciden en la calidad de la obturación. Dentro de estos factores se encuentran, la inexperiencia de los estudiantes, la anatomía del sistema de conductos, factores propios del paciente, entre otros (Lynch \& Burke, 2006).

\footnotetext{
${ }^{1}$ Escuela de Odontología, Facultad de Medicina, Universidad Austral de Chile, Valdivia, Chile.

2 Profesor Adjunto, Instituto de Odontoestomatología, Facultad de Medicina, Universidad Austral de Chile, Valdivia, Chile.

${ }^{3}$ Profesor Auxiliar, Instituto de Odontoestomatología, Facultad de Medicina, Universidad Austral de Chile, Valdivia, Chile.
} 
Según Gilbert et al. (2010) el éxito del tratamiento endodóntico, se puede evaluar por hallazgos radiológicos o clínicos por sí solos, o ambos en conjunto. La evaluación radiográfica representa un método de evaluación muy frecuente ( $\mathrm{Ng}$ et al., 2010), los parámetros técnicos de calidad radiográfica generalmente evaluados son la longitud del material de relleno en relación con el ápice radiográfico, la densidad del material de relleno y, por último, la incidencia de errores de procedimiento, por ejemplo, transporte, escalón, perforación o fractura de instrumentos (Ribeiro et al., 2018). Además, para obtener éxito en el tratamiento endodóntico, es importante la eliminación de los microorganismos en los conductos radiculares y el cuidado del tejido periapical, por lo tanto, el resultado clínico del tratamiento se debe clasificar en términos de éxito, cuando en el examen de control no hay signos ni síntomas clínicos (ausencia de dolor e inflamación, función normal del diente) o radiolucencia periapical (desaparición o reducción de la lesión o la rarefacción ósea periapical) (Friedman, 2002).

El éxito radiográfico ha sido descrito en un metaanálisis de Ribeiro et al., entre los años 1995-2015, el cual fue realizado en estudiantes de pregrado; se obtuvo una frecuencia de éxito que varió de un $13 \%$ a un $85 \%$, con una frecuencia promedio de $48,75 \%$ de calidad aceptable de las obturaciones radiculares. A su vez, Khabbaz et al. (2010) reveló un porcentaje de éxito radiográfico en dentistas generales como especialistas, que varía desde un $14 \%$ a $56,9 \%$ en dentistas generales y de $91 \%$ en especialistas respectivamente. En tanto, el éxito clínico de tratamientos realizados por estudiantes de pregrado fue descrito por Valera et al. (2012) en Brasil, donde se obtuvo una alta tasa clínica de un 89,36 \%. En Chile, una investigación realizada por Alcota Rojas \& Zepeda Zárate (2010) arrojó un $57 \%$ de éxito radiográfico, sin embargo, este estudio solo fue realizado en estudiantes de postgrado de la especialidad de Endodoncia.

A nivel nacional existe un número limitado de investigaciones relacionadas con el éxito radiográfico y/o clínico, algunos de estos estudios fueron realizados en estudiantes de postgrado y abarcando sólo éxito radiográfico (Alcota Rojas \& Zepeda Zárate). Por lo tanto, son necesarios estudios que evalúen tanto el éxito clínico y radiográfico; particularmente en estudiantes de pregrado a nivel nacional para identificar fortalezas y debilidades en los procedimientos, y así también generar cambios a nivel teórico-práctico, en caso de ser necesarios, con el fin de generar mejoras en los pronósticos de estos tratamientos.
El objetivo general de este estudio fue evaluar y determinar el éxito clínico/radiográfico en los tratamientos endodónticos realizados en pacientes atendidos en la clínica de pregrado de la Universidad Austral de Chile (UACh) entre los años 2014-2017.

\section{MATERIAL Y MÉTODO}

Se realizó un estudio observacional descriptivo a través de los datos recogidos del registro de pacientes que se realizaron tratamientos endodónticos entre los años 2014 y 2017, efectuado por estudiantes de pregrado de la escuela de odontología de la UACh, Valdivia, Chile. Este estudio contó con la aprobación del Comité Ético Científico del Servicio de Salud de Valdivia ( $\left.N^{\circ} 1349375\right)$, Chile, y fue autorizado por el Instituto de Odontoestomatología de la UACh.

Los criterios de inclusión son: presencia de radiografía previa en buenas condiciones, radiografía de control sin errores técnicos ni de exposición y dientes de pacientes mayores de 18 años; criterios de exclusión: documentación incompleta, tratamientos sin finalizar, endodoncias sin tratamiento rehabilitador, dientes con prótesis fija unitaria (PFU) con anclaje intrarradicular, retratamientos endodónticos, dientes birradiculares, dientes que fueron sometidos a clareamiento interno, dientes con curvatura apical muy pronunciada y obturaciones radiculares realizadas con agregado de trióxido mineral (MTA).

De los 1.727 tratamientos endodónticos realizados entre los años 2014-2017, 286 fueron seleccionadas al aplicar los criterios de inclusión/exclusión, a partir de esta cifra se calculó el tamaño muestral mediante Epidat (versión 4.2, julio 2016), dando como resultado 159 dientes tratados y se agregó una pérdida del $10 \%$ de pacientes estimando una muestra de 175 , la cual fue escogida mediante muestreo aleatorio simple. Luego, los pacientes que accedieron voluntariamente a participar fueron informados del estudio y firmaron un consentimiento informado previo a la evaluación, con el fin de realizar un nuevo examen radiográfico control estandarizado, la evaluación radiográfica y clínica actual. La muestra final correspondió a 74 tratamientos endodónticos en 63 pacientes.

La pauta de evaluación consistió en un área de evaluación radiográfica y clínica. En el área radiográfica, se evaluó las radiografías de control postoperatorias estandarizadas las cuales fueron eva- 
luadas por evaluadores calibrados previamente (calibración intra e interobservador- índice Kappa superior a 0,8 ), se observó la calidad de obturación radicular de acuerdo con los criterios descritos por BarrieshiNusair et al. (2004) basado en el consenso de la Sociedad Europea de Endodoncia, que incluyen longitud de obturación, densidad, conicidad y errores procedimentales. Si todos estos criterios eran adecuados, se clasificaba como éxito radiográfico. Cualquier alteración en estos criterios, se clasificaba como fracaso radiográfico. En el área clínica se evaluó la ausencia de dolor a la percusión, ausencia de dolor en palpación de fondo de vestíbulo, ausencia de aumento de volumen, ausencia de fístula y diente en función. Si todos los criterios eran adecuados, se consignaba como éxito clínico. Cualquier alteración en estos criterios, se consignaba como fracaso clínico.

Se registró, además, el tipo de diente, grupo etario, sexo del paciente y diagnóstico pulpar y periapical.

Los datos fueron ingresados en una hoja de cálculo de Google Sheets de acceso limitado a los autores del estudio, luego se realizó un análisis descriptivo de los datos ingresados.

Tabla I. Distribución según grupo etario de los tratamientos endodónticos

\begin{tabular}{cl}
\hline Grupo etario & Frecuencia \\
\hline $18-30$ & $12(16,2 \%)$ \\
$31-40$ & $7(9,4 \%)$ \\
$41-60$ & $36(48,7 \%)$ \\
$60>$ & $19(25,7 \%)$ \\
Total & $74(100 \%)$ \\
\hline
\end{tabular}

\section{RESULTADOS}

Se evaluaron 63 pacientes, con un total de 74 tratamientos endodónticos de los cuales 48 (65\%) fueron en mujeres y 26 (35\%) fueron realizados en hombres.

Los dientes pertenecientes a la arcada dental maxilar fueron los más tratados endodónticamente con 50 dientes $(67,57 \%$ ) y en la arcada mandibular 24 (32.43 $\%)$. Los dientes anteriores fueron 46 , lo que corresponde a un $64,9 \%$ y 28 fueron premolares $(35,1 \%)$. El 59 $\%$ del total de los tratamientos realizados fueron realizados en 2 sesiones. La distribución por grupo etario se observa en la Tabla I.

El éxito radiográfico alcanzado fue de un $41 \%$ y el éxito clínico fue de un $78 \%$, además, el número de tratamientos que presentaron tanto éxito radiográfico como clínico fue de un $36 \%$ (Tabla II).

Pulpitis irreversible sintomática y necrosis pulpar fueron los diagnósticos pulpares más frecuentes en los tratamientos realizados; en cuanto a diagnósticos apicales los más frecuentes fueron periodontitis apical sintomática y tejido apical normal (Tabla III).

En las Tablas IV y $\mathrm{V}$ se muestra el detalle de la evaluación clínica y radiográfica de los tratamientos en la actualidad; en relación a la evaluación radiográfica, los tratamientos obtuvieron un nivel óptimo en longitud en un $83,8 \%, 52,7 \%$ en densidad y $85,1 \%$ en conicidad. En el aspecto clínico, se observaron 60 tratamientos con

Tabla II. Frecuencia de éxito clínico y radiográfico.

\begin{tabular}{lccc}
\hline & Clínico & Radiográfico & Clínico \& Radiográfico \\
\hline Éxito & $58(78 \%)$ & $30(41 \%)$ & $27(36 \%)$ \\
Fracaso & $16(22 \%)$ & $44(59 \%)$ & $13(18 \%)$ \\
\hline
\end{tabular}

Tabla III. Frecuencias de diagnósticos pulpares y apicales previo al tratamiento endodóntico.

\begin{tabular}{lclc}
\hline Diagnóstico Pulpar & Frecuencia & Diagnóstico apical & Frecuencia \\
\hline Pulpa normal & $3(4,1 \%)$ & Tejido apical normal & $26(35,1 \%)$ \\
Pulpitis reversible & $3(4,1 \%)$ & Periodontitis apical sintomática & $32(43,2 \%)$ \\
Pulpitis Irreversible sintomática & $22(29,7 \%)$ & Periodontitis apical asintomática & $9(12,2 \%)$ \\
Pulpitis irreversible asintomática & $14(18,9 \%)$ & Absceso apical agudo & $1(1,4 \%)$ \\
Necrosis pulpar & $27(36,5 \%)$ & Absceso apical crónico & $6(8,1 \%)$ \\
Terapia previamente iniciada & $5(6,8 \%)$ & & \\
\hline
\end{tabular}

Tabla IV. Distribución de los parámetros clínicos evaluados.

\begin{tabular}{lcccc} 
& Dolor & Aumento de volumen & Fístula & Diente en función \\
\hline Presente & $14(18,9 \%)$ & $0(0 \%)$ & $0(0 \%)$ & $72(97,3 \%)$ \\
Ausente & $60(81,1 \%)$ & $74(100 \%)$ & $74(100 \%)$ & $2(2,7 \%)$ \\
\hline
\end{tabular}


ausencia de dolor (81,08\%), un $97 \%$ de los dientes intervenidos estaban en función, y no existieron casos con fístula ni aumento de volumen.
Los únicos errores de procedimiento reportados y observados fueron escalón (2,7 \%) y falsa vía (5 \%).

Tabla V. Distribución de los parámetros radiográficos evaluados.

\begin{tabular}{llll}
\hline & Longitud & Densidad & Conicidad \\
\hline Adecuado & $62(83,8 \%)$ & $39(52,7 \%)$ & $63(85,1 \%)$ \\
Inadecuado & $12(16,2 \%)$ & $35(47,3 \%)$ & $11(14,9 \%)$ \\
\hline
\end{tabular}

\section{DISCUSIÓN}

En el ámbito radiográfico, un metaanálisis realizado en estudiantes de pregrado por Ribeiro et al. alcanzó un éxito radiográfico del $39 \%$ al $57 \%$ con promedio de 48,75\%. El estudio realizado por Khabbaz et al. obtuvo una frecuencia de éxito radiográfico en estudiantes de pregrado del $55 \%$ y un estudio realizado en una universidad chilena (Alcota Rojas \& Zepeda Zárate) obtuvo un éxito radiográfico de un 57 $\%$ en tratamientos realizados con estudiantes de postgrado. A nivel clínico, una investigación realizada en estudiantes de pregrado por Valera et al. reflejó un $89,36 \%$ de éxito. Las tasas de éxito radiográfico y clínico nombradas anteriormente coinciden con los resultados obtenidos en el presente estudio, de un $41 \%$ y $78 \%$, respectivamente.

En lo que se refiere a los parámetros de la evaluación radiográfica, los tratamientos endodónticos evaluados en el presente trabajo presentan una frecuencia adecuada de longitud de un $83 \%$, densidad $52 \%$ y conicidad $85 \%$; Khabbaz et al. determinó una longitud aceptable de $54,8 \%$, resultado inferior al obtenido en el presente estudio y densidad aceptable de $66,5 \%$, lo que es superior a nuestros resultados respectivamente.

Un estudio que obtuvo resultados similares al nuestro fue el de Alcota Rojas \& Zepeda Zárate con longitud aceptable de $86,6 \%$ y densidad $61,7 \%$. En cuanto a conicidad, la única investigación que presenta una evaluación coincidente en este parámetro fue de Barrieshi-Nusair et al. con un 85,3\%, mientras que los resultados de Eleftheriadis \& Lambrianidis (2005) son $62,7 \%$ en longitud y $82,6 \%$ en densidad.

En cuanto, a los datos de fracasos clínicos reportado por Valera et al. lo más frecuente informado por los pacientes fue dolor (93,75\% de los casos), y en el $43,75 \%$ de estos casos el dolor se asoció con la presencia de fístula y/o edema.
En cuanto a la mayor tasa de éxito en anteriores que premolares, esto se puede deber a que los dientes anteriores generalmente tienen un solo canal que conduce al ápice con un menor grado de curvatura, lo que puede contribuir a mejores resultados (Ilgüy et al., 2013).

Algunos autores han informado que cuanto menos densa y homogénea sea la obturación radicular, mayor será la probabilidad de un impacto negativo en el resultado del tratamiento (Kerekes \& Tronstad 1979, Stoll et al. 2005). Además, las obturaciones con una masa homogénea sin vacíos están fuertemente correlacionados con mejores resultados (Hayes et al., 2001). Siqueira Jr. (2001) afirma que la desinfección del sistema de conductos radiculares no se puede determinar a partir de las radiografías y, como consecuencia, incluso los canales aparentemente bien obturados pueden permanecer infectados. Asimismo, numerosos estudios de países europeos han demostrado que un alto porcentaje de dientes adecuadamente obturados persisten con periodontitis apical (Eriksen \& Bjertness, 1991; Loftus et al., 2005; Sunay et al., 2007).

Otro punto a destacar es que las evaluaciones de imágenes se limitaron a las radiografías intraorales, que proporcionan vistas bidimensionales (2D), que carecen de la dimensión bucal-palatina y, posiblemente, enmascaran los errores de las obturaciones radiculares en este plano mediante la superposición de estructuras anatómicas. Actualmente, el uso de imágenes de tomografía computarizada de haz cónico (CBCT), ha permitido una mayor precisión y resolución en la evaluación de las estructuras anatómicas en 3D (Special Committee to Revise the Joint AAE/AAOMR Position Statement on use of CBCT in Endodontics, 2015).

Los resultados heterogéneos encontrados en el metanálisis (Ribeiro et al.) se puede explicar por una 
falta de estandarización en el plan de estudios de las escuelas de odontología, el nivel de los estudiantes, las técnicas de endodoncia y la región del diente tratado, lo cual pueden tener un impacto en la calidad técnica de las obturaciones de las raíces evaluadas, como de los resultados clínicos obtenidos. En este sentido, examinar el trabajo realizado por los estudiantes con regularidad es una manera de asegurar si se está brindando un estándar de tratamiento adecuado (Donnelly et al., 2017).

Existen varias maneras de evaluar un tratamiento endodóntico, sin embargo, aún no existe un consenso en los criterios de éxito y fracaso. La mayoría de los autores concuerdan en afirmar que la determinación del resultado del diente tratado, estará dada por la evaluación integral clínica y radiográfica, y que, a su vez, debe presentarse una combinación de normalidad clínica y radiográfica para considerar el caso exitoso (Sankarsingh Morillo, 2003). En el presente estudio, se realizó una estricta evaluación tanto de los parámetros clínicos y radiográficos. En particular, criterios radiográficos como la presencia de un pequeño poro, oquedad o espacio vacío en la obturación radicular detectado en cualquier nivel de la obturación radicular, se clasificaban como fracaso radiográfico, a pesar, que en muchas oportunidades estos iban de la mano del éxito clínico o ausencia de cualquier sintomatología y con un diente perfectamente funcional. Esta estricta evaluación puede explicar los resultados obtenidos en este punto.

Una de las limitantes del estudio, fue el hallazgo de fichas con exámenes radiológicos de pobre calidad de los tratamientos realizados, lo que provocó, además, la disminución del tamaño muestral.

En conclusión, el tratamiento endodóntico realizado por los estudiantes de pregrado de la Escuela de Odontología de la UACh mostró una tasa de éxito radiográfico y clínico de $41 \%$ y $78 \%$, respectivamente. Generalmente, el éxito radiográfico siempre será menor al éxito clínico, por ende, se recomienda evaluar el tratamiento endodóntico con ambos criterios y a su vez realizar un seguimiento periódico, según cada caso.

Este estudio presenta gran importancia clínica, ya que permite encontrar falencias y crear mejoras en la práctica odontológica a nivel de pregrado. Las asignaturas preclínicas son los principales contribuyentes en este proceso de mejora en la parte técnica de los tratamientos, por lo que es importante conocer y eva- luar la calidad de los procedimientos realizados por los estudiantes de pregrado.

Se sugiere realizar nuevos estudios prospectivos para hacer un seguimiento a los tratamientos endodónticos y establecer asociación entre las variables, incluir dientes birradiculares y utilizar otras técnicas imagenológicas.

MALDONADO-SANHUEZA, F.; GÓMEZ-INZUNZA, V.; ROSAS-MENDEZ, C. \& HERNÁNDEZ-VIGUERAS, S. Evaluation of success rate of root canal treatment performed by undergraduate dental students in a Chilean university. Int. J. Odontostomat., 14(2):154-159, 2020.

ABSTRACT: The objective of endodontic treatment is to prevent or achieve the absence of post-treatment apical periodontitis, thereby maintaining functionality of the tooth in the mouth. In Chilean universities undergraduate programs, training in endodontics is limited to low level complexity cases and is transversal throughout the universities. The objective of this study is to evaluate the frequency of success of endodontic treatments performed by undergraduate students between the years 2014-2017 of the School of Dentistry, Universidad Austral de Chile (UACh), Valdivia, Chile. A descriptive observational study was conducted where 74 endodontic treatments were evaluated in uniradicular teeth of 63 patients, selected through a simple random sampling, that met selection criteria. The criteria used for the clinical evaluation were: absence of pain on palpation and percussion, absence of fistula and function tooth. In the radiographic evaluation the following criteria were used: length of obturation in relation to the dental apex, taper, density of the obturator material and reduction of the periapical lesion. Of the treatments evaluated, $78 \%$ clinical success and $41 \%$ radiographic success were reported. Finally, it is concluded that the results obtained at clinical and radiographic level are similar to those reported in the literature.

KEY WORDS: endodontic, root canal obturation, root canal filling materials, radiography dental, pain, postoperative, dental students, success, failure.

\section{REFERENCIAS BIBLIOGRÁFICAS}

Alcota Rojas, M. \& Zepeda Zárate, C. Calidad técnica de la obturación radiográfica de tratamientos realizados por estudiantes del posgrado en Endodoncia de la Universidad de Chile. Endodoncia, 28(4):215-9, 2010.

American Association of Endodontists. Appropriateness of Care and Quality Assurance Guidelines. Chicago, American Association of Endodontists, 1998. 
Barrieshi-Nusair, K. M.; Al-Omari, M. A. \&Al-Hiyasat, A. S. Radiographic technical quality of root canal treatment performed by dental students at the dental teaching center in Jordan. J. Dent., 32(4):3017, 2004.

Donnelly, A.; Coffey, D. \& Duncan, H. F. A re-audit of the technical quality of undergraduate root canal treatment after the introduction of new technology and teaching practices. Int. Endod. J., 50(10):941-50, 2017.

Eleftheriadis, G. I. \& Lambrianidis, T. P. Technical quality of root canal treatment and detection of iatrogenic errors in an undergraduate dental clinic. Int. Endod. J., 38(10):725-34, 2005.

Eriksen, H. M. \& Bjertness, E. Prevalence of apical periodontitis and results of endodontic treatment in middle-aged adults in Norway. Endod. Dent. Traumatol., 7(1):1-4, 1991.

Friedman, S. Considerations and concepts of case selection in the management of post-treatment endodontic disease (treatment failure). Endod. Top., 1(1):54-78, 2002.

Gilbert, G. H.; Tilashalski, K. R.; Litaker, M. S.; McNeal, S. F.; Boykin, M. J.; Kessler, A. W. \& DPBRN Collaborative Group. Outcomes of root canal treatment in dental practice-based research network practices. Gen. Dent., 58(1):28-36, 2010.

Hayes, S. J.; Gibson, M.; Hammond, M.; Bryant, S. T. \& Dummer, P. $M$. An audit of root canal treatment performed by undergraduate students. Int. Endod. J., 34(7):501-5, 2001.

Ilgüy, D.; Ilgüy, M.; Fisekçioglu, E.; Ersan, N.; Tanalp, J. \& Dölekoglu, S. Assessment of root canal treatment outcomes performed by Turkish dental students: results after two years. J. Dent. Educ., 77(4):502-9, 2013.

Khabbaz, M. G.; Protogerou, E. \& Douka, E. Radiographic quality of root fillings performed by undergraduate students. Int. Endod. J., 43(6):499-508, 2010.

Loftus, J. J.; Keating, A. P. \& McCartan, B. E. Periapical status and quality of endodontic treatment in an adult Irish population. Int. Endod. J., 38(2):81-6, 2005.

Lynch, C. D. \& Burke, F. M. Quality of root canal fillings performed by undergraduate dental students on single-rooted teeth. Eur. J. Dent. Educ., 10(2):67-72, 2006.

Marquis, V. L.; Dao, T.; Farzaneh, M.; Abitbol, S. \& Friedman, S. Treatment outcome in endodontics: the Toronto Study. Phase III: initial treatment. J. Endod., 32(4):299-306, 2006.

$\mathrm{Ng}$, Y. L.; Mann, V. \& Gulabivala, K. Tooth survival following non-surgical root canal treatment: a systematic review of the literature. Int. Endod. J., 43(3):171-89, 2010.

Ray, H. A. \& Trope, M. Periapical status of endodontically treated teeth in relation to the technical quality of the root filling and the coronal restoration. Int. Endod. J., 28(1):12-8, 1995.

Ribeiro, D. M.; Réus, J. C.; Felippe, W. T.; Pacheco-Pereira, C.; Dutra, K. L.; Santos, J. N.; Porporatti, A. L. \& De Luca Canto, G. Technical quality of root canal treatment performed by undergraduate students using hand instrumentation: a meta-analysis. Int. Endod. J., 51(3):269-83, 2018.

Sankarsingh Morillo, C. C. Determinación de Éxito y Fracaso en el Tratamiento de Conductos. En: Bóveda Z., C. El Odontólogo Invitado. Sitio Web. Caracas, Carlos Bóveda, 2003. Disponible: https:/ /www.carlosboveda.com/Odontologosfolder/odontoinvitadoold/ odontoinvitado_33.htm

Siqueira Jr., J. F. Aetiology of root canal treatment failure: why welltreated teeth can fail. Int. Endod. J., 34(1):1-10, 2001.

Sjogren, U.; Hagglund, B.; Sundqvist, G. \& Wing, K. Factors affecting the long-term results of endodontic treatment. J. Endod., 16(10):498-504, 1990.

Special Committee to Revise the Joint AAE/AAOMR Position Statement on use of CBCT in Endodontics. AAE and AAOMR Joint Position Statement: Use of Cone Beam Computed Tomography in Endodontics 2015 Update. Oral Surg. Oral Med. Oral Pathol. Oral Radiol., 120(4):508-12, 2015.
Sunay, H.; Tanalp, J.; Dikbas, I. \& Bayirli, G. Cross-sectional evaluation of the periapical status and quality of root canal treatment in a selected population of urban Turkish adults. Int. Endod. J., 40(2):139-45, 2007.

Valera, M. C.; Araújo, M. A. M.; Fernandes, A. M.; Camargo, C. H. R. \& Carvalho, C. A. T. Assessment of success rate of endodontic treatment performed by Brazilian undergraduate students. Dent. Press Endod., 2(2):25-9, 2012.

Wu, M. K.; Shemesh, H. \& Wesselink, P. R. Limitations of previously published systematic reviews evaluating the outcome of endodontic treatment. Int. Endod. J., 42(8):656-66, 2009.

Dirección para correspondencia:

Scarlette Hernández-Vigueras.

Instituto de Odontoestomatología

Rudloff 1640

Valdivia

CHILE

Email: shernandez@uach.cl

Recibido : 13-08-2019

Aceptado: 10-10-2019 